\title{
Experimental validation of the reverse polar decomposition of depolarizing Mueller matrices
}

\author{
Makrina Anastasiadou \\ makrina.anastasiadou@polytechnique.edu
}

\section{Sami Ben Hatit}

\section{Razvigor Ossikovski}

\section{Steve Guyot}

\section{Antonello De Martino}

\author{
LPICM, Ecole Polytechnique, CNRS, 91128 Palaiseau, France
}

LPICM, Ecole Polytechnique, CNRS, 91128 Palaiseau, France

LPICM, Ecole Polytechnique, CNRS, 91128 Palaiseau, France

LPICM, Ecole Polytechnique, CNRS, 91128 Palaiseau, France

We experimentally assess the validity of the reverse polar decomposition (R. Ossikovski et al., Opt. Lett. 32, 689 (2007)), which describes any Mueller matrix as a product of a depolarizer, a diattenuator and a retarder with the diattenuator placed after the depolarizer and not before, as in the well-known Lu and Chipman's forward decomposition. The raw data are Mueller images of a depolarizer (dilute milk at variable concentrations), followed by two tilted glass plates as a diattenuator and a mica retardation plate. While the reverse decomposition accurately reconstructs the component matrices in all cases, the usual forward decomposition provides reasonable values only for the trace of the depolarizer matrix, the other quantities being affected by gross errors. The potential interest of this decomposition for biological samples is briefly discussed. [DOI: 10.2971/jeos.2007.07018]

Keywords: Polar decomposition, reverse, Mueller matrix, polarization, dichroism

\section{INTRODUCTION}

Ten years ago Lu and Chipman [1] proposed a three-factor matrix decomposition of any physical Mueller matrix $\mathbf{M}$ based on a generalization of the polar decomposition to the depolarizing case. Since then, this decomposition comprising a diattenuator $\mathbf{M}_{D}$, a retarder $\mathbf{M}_{R}$ and a depolarizer $\mathbf{M}_{\Delta}$ has been widely used in the interpretation of experimental Mueller matrices and, in particular, in imaging polarimetry [2]-[4]. More specifically, the initial matrix $\mathbf{M}$ is typically cast as:

$$
\mathbf{M}=\mathbf{M}_{\Delta} \mathbf{M}_{R} \mathbf{M}_{D}
$$

where the diattenuator matrix $\mathbf{M}_{D}$ is defined directly from the dichroism vector $\mathbf{D}$ of $\mathbf{M}$ as

$$
\mathbf{M}_{D}=m_{11}\left(\begin{array}{cc}
1 & \mathbf{D}^{T} \\
\mathbf{D} & \mathbf{m}_{D}
\end{array}\right)
$$

where the submatrix $\mathbf{m}_{D}$ has the form defined in Ref. [1], the retarder matrix has the form

$$
\mathbf{M}_{R}=\left[\begin{array}{cc}
1 & \overrightarrow{0}^{T} \\
\overrightarrow{0} & \mathbf{m}_{R}
\end{array}\right]
$$

where $\mathbf{m}_{R}$ is a three dimensional rotation matrix, and the depolarizer matrix

$$
\mathbf{M}_{\Delta}=\left[\begin{array}{cc}
1 & \overrightarrow{0}^{T} \\
\vec{P}_{\Delta} & \mathbf{m}_{\Delta}
\end{array}\right]
$$

is characterized by zero dichroism and generally nonzero polarizance (the submatrix $\mathbf{m}_{\Delta}$ is symmetric and its precise expression is also given in Ref. [1]). As discussed in Ref. [5] the other two decompositions with the depolarizer set after the diattenuator are readily deduced from Eq. (1) and form a first family of "forward decompositions".

On the other hand, the three decompositions with the depolarizer set before the diattenuator, which form the "reverse" family, may lead to unphysical component matrices, or may even not exist, if the three components are assumed to be of the form described by Eqs. (2-4). This issue has been solved recently with the reverse polar decomposition [6] for which one of the three possible forms is:

$$
\mathbf{M}=\mathbf{M}_{R}^{r} \mathbf{M}_{D}^{r} \mathbf{M}_{\Delta}^{r}
$$

where the dichroism of the diattenuator is now taken equal to the polarizance $\overrightarrow{\mathbf{P}}$ of the initial matrix $\mathbf{M}$

$$
M_{D}^{r}=m_{11}\left(\begin{array}{cc}
1 & \vec{P}^{T} \\
\vec{P} & m_{P}
\end{array}\right)
$$

and the depolarizer is of a slightly different type, with zero polarizance and non zero dichroism :

$$
M_{\Delta}^{r}=\left[\begin{array}{cc}
1 & \vec{D}^{T} \\
\overrightarrow{0} & m_{\Delta}
\end{array}\right]
$$

The physical basis of this reverse decomposition is easily understood in the simple case of a perfect depolarizer combined 
with a diattenuator. If the diattenuator is traversed first, as assumed in the forward decomposition, then the compound system will exhibit zero polarizance and a dichroism equal to that of the diattenuator; in the opposite case, where the depolarizer comes first, the compound system will exhibit zero dichroism while the dichroism of the diattenuator will be simply equal to the polarizance of the whole system.

The purpose of this paper is to provide an experimental investigation of the robustness and the accuracy of this reverse decomposition with Mueller matrices measured on a system comprising a variable depolarizer followed by a diattenuator and a retarder. The experimental setup, the samples and the measurement procedures are briefly described in section 2. Section 3 is devoted to the results of both decompositions of the measured polarimetric images. The potential usefulness of the reverse decomposition in biomedical applications is also discussed from an example of Mueller image of a biological tissue sample. Section 4 concludes the paper.

\section{EXPERIMENTAL}

\subsection{The imaging Mueller polarimeter in reflection}

Our Mueller imaging polarimeter was an upgraded version of the instrument described in Ref. [3] and is outlined in Figure 1. More details will be given in a forthcoming publication.

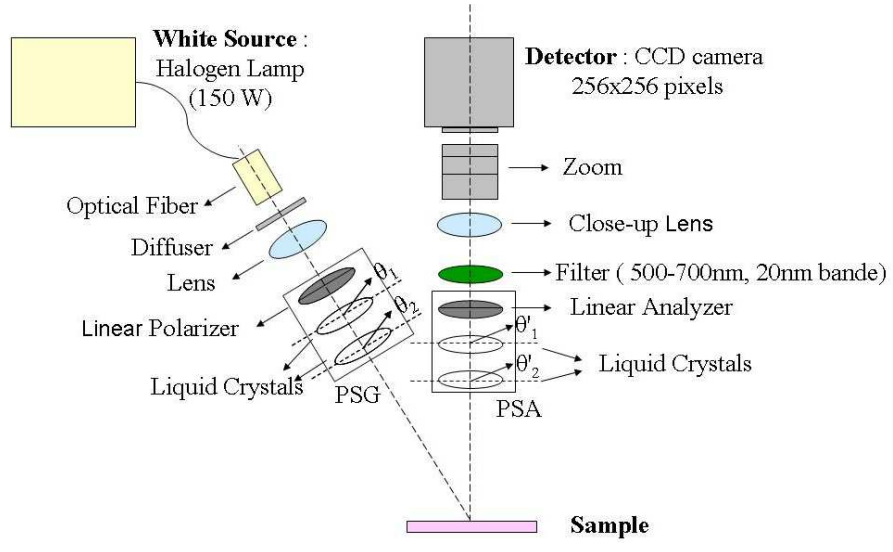

FIG. 1 Scheme of the imaging Mueller polarimeter in reflection.

The illumination system comprised a halogen lamp (Olympus CLH-SC 150 W), a fiber bundle, a diffuser (Newport Light Shaping Diffuser $10^{\circ}$ ) and an aspherical condenser (Newport KPA046, $f=37 \mathrm{~mm}$ ). This system provided a reasonably homogenous illumination of the field of view up to about $4 \mathrm{~cm}$ diameter.

The Polarisation State Generator (PSG) was developed according to the optimized design described in Ref. [7], with a linear polarizer (Melles Griot, 03 FPG 007) followed by two nematic liquid crystal variable retarders (Meadowlark LVR 300). This PSG sequentially generated four polarization states by alternatively switching each liquid crystal between two suitably chosen retardations. The Polarization State Analyzer (PSA), which analyzed the polarization of the light emerging from the sample, comprised the same elements as those of the PSG, but positioned in reverse order. As a result, this PSA was also optimized with the same operating parameters as the PSG.

The sample was imaged on a fast CCD camera (Dalsa CAD1, 256x256 pixels, 12 bits) by means of a 12.5-75 $\mathrm{mm}$ zoom with an additional $500 \mathrm{~mm}$ close-up lens. The operation wavelength could be selected between 500 and $700 \mathrm{~nm}$ in steps of $50 \mathrm{~nm}$ by means of interference filters (20 nm spectral bandwidth) directly mounted on the close-up. This arrangement allowed an efficient rejection of any stray light.

The dark current, which depended on the room temperature was measured frequently and subtracted from the signal for each pixel. A satisfactory signal-to-noise ratio was achieved by typically averaging 7 images for each state of polarization. A complete set of 16 averaged raw images was taken in about 11 seconds.

The polarimeter was calibrated by a procedure quite similar to that described in Ref. [3], adapted to the operation in reflection. With this procedure, the Mueller matrix of a mirror is the unit matrix, as for propagation in vacuum. With respect to the more usual convention the signs of the last two rows of the Mueller matrices are changed. We retained this choice as it greatly facilitates the visual comparison of the $m_{22}$ and $m_{33}$ matrix elements, which are then expected to be equal and not opposite.

\subsection{The samples}

The samples imaged in this study are outlined on Figure 2, and consisted of a spatially inhomogeneous depolarizer, a diattenuator and a retarder, traversed by the light beam in this order.
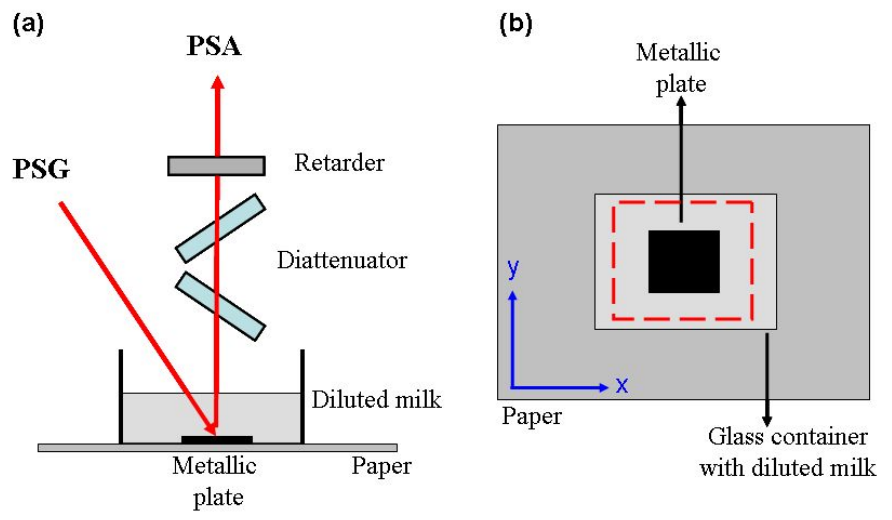

FIG. 2 Experimental set-up containing a spatially inhomogeneous depolarizer (metallic plate at the bottom of a container with diluted milk), followed by a diattenuator (two tilted glass plates), followed by a retarder (mica quarter wave-plate). (a): side view of the entire setup. (b) view from the top of the depolarizer. The red dotted line on Figure (b) defines the field imaged on the CCD.

The retarder was a commercially available $50 \mathrm{~mm}$ clear aperture mica quarter wave plate (Melles Griot 02WRM009). This element also can be inserted and removed without any displacement of the image of the depolarizer. 
The diattenuator consisted of two high index $(n=1.8)$ glass plates, tilted as shown on the Figure 2 at the highest possible incidence (close to $45^{\circ}$ ) compatible with a complete coverage of the field, with a uniform diattenuation close to 0.3 . This diattenuation was kept constant throughout this investigation (it could be easily decreased by reducing the tilt angle). This diattenuator could be inserted and removed without any significant displacement of the image.

As a spatially inhomogeneous controllable depolarizer we used a transparent glass container, resting on a white piece of paper, with a small square metallic plate at the center of the field of view. This container was filled with milk diluted in water at variable concentrations. As explained below, the outer part of the image, corresponding to the paper seen through the solution and the bottom of the glass container, always exhibited strong depolarization, while for the plate at the image center the depolarization could be adjusted from 0 to 1 by changing the milk concentration.

Depolarization always involves incoherent intensity superposition of light beams with different polarizations. Superposition of many light wave contributions with different polarizations naturally occurs with samples featuring bulk scattering: the photons emerging at any given point of the sample come from different locations in the incident beam, through different scattering paths leading to different polarizations even for a uniformly polarized illumination. If the incident beam is spatially coherent, as it is typically the case for most low power cw lasers, this superposition leads to speckle patterns with spatially random elliptical polarizations. The time evolution of these patterns is directly related to that of the sample, and may be measured and used, for example, for particle size determination [8]. The observed depolarization then depends on whether the integration time of the detection is longer or shorter than the speckle pattern evolution time. In contrast, with typical classical sources - with a geometrical extension well above the diffraction limit - the incident beam can be considered as coherent over times of the order of the reciprocal of the optical bandwidth, typically picoseconds or shorter. As a result, the "instantaneous" speckle patterns are modified in times of this order, leading to intensity averaging and depolarization even for static samples, and independently of detector time response.

As our setup was illuminated with a classical extended source, the paper always appeared as highly depolarizing due to its bulk scattering properties. In contrast, the plate exhibited essentially surface scattering, without any significant light penetration into the bulk. As the superposition of different light contributions outlined above could not occur in this case the plate itself appeared as essentially nondepolarizing. Of course, this was no longer true when milk was added due to the light scattering taking place in the solution above the plate.

The measurements were performed as follows: the container was filled with $30 \mathrm{ml}$ of water first, and milk concentration was gradually increased, from 0 to $5 \%$. For each concentration, three Mueller images were taken, the first one with the depolarizer alone, the second one with the depolarizer fol- lowed by the diattenuator and the third one with the retarder added to the other two components.

Figure 3 shows typical experimental Mueller matrices, taken at $0.6 \%$ milk concentration. The top image, taken with the depolarizer alone, exhibits the expected shape for a suspension of spherical scatterers, i.e. diagonal Mueller matrices with equal values of $m_{22}$ and $m_{33}$. As expected, the central part of the image, corresponding to the metal plate, features higher values of the diagonal terms than the surrounding part, corresponding to the paper.

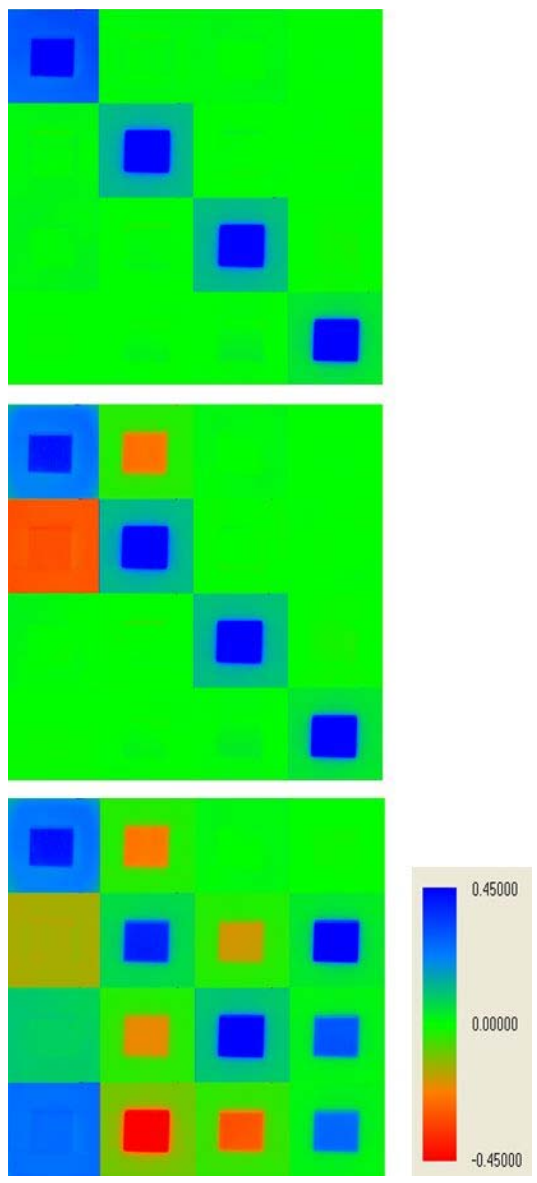

FIG. 3 Mueller matrix images taken with $0.6 \%$ milk concentration. Top: depolarizer alone. Middle: depolarizer and diattenuator. Bottom: depolarizer, diattenuator and retarder. All elements but the unpolarized reflectivity $m_{11}$ are normalized by $m_{11}$ and displayed according to the colorscale shown at the bottom right.

For the middle image, the diattenuator was included, with its axes aligned with the reference axes of the Stokes and Mueller matrices (the $x$ and $y$ directions on the right panel of the Figure 2). Again, the measured Mueller images exhibit the expected trends: with respect to the previous image, only the $m_{12}$ and $m_{21}$ are affected. Morevoer, the observed diattenuation $\left(m_{12}\right)$ is significant only on the metal plate, and practically vanishes in the peripheral part due the strong depolarization characteristic of the paper. In contrast, the polarizance $\left(m_{21}\right)$ is uniform, as expected for a diattenuator set after the depolarizer and covering the whole field of view.

Finally, in the bottom image taken with the retarder added after the diattenuator, the elements of the lower $3 \times 3$ block are mixed by the rotation matrix $\mathbf{m}_{R}$ describing the retarder. 


\subsection{The data analysis}

We apply the forward and reverse decompositions to every measured Mueller matrix image and we evaluate the accuracy of the resulting depolarizer $\left(\mathbf{M}_{\Delta}\right)$, diattenuator $\left(\mathbf{M}_{D}\right)$ and retarder $\left(\mathbf{M}_{R}\right)$ by comparison with independent data on these matrices. More specifically, the quality of the "reconstruction" of the diattenuator and the retarder provided by these decompositions is assessed from the constancy of the scalar diattenuation $D$ and the retardation $R$ throughout each image, and when the milk concentration is varied.

The accuracy of $\mathbf{M}_{\Delta}$ is evaluated in more detail, by comparison with the measurements carried out with the depolarizer alone. The depolarization power of a system described by a Mueller matrix $\mathbf{M}$ is typically evaluated by two indicators:

- The average depolarization [9], easily applicable to any Mueller matrix and defined by

$$
\Delta_{M}=1-\left[\frac{\sum_{i j=1}^{4} m_{i j}^{2}-m_{11}^{2}}{3 m_{11}^{2}}\right]^{\frac{1}{2}}
$$

- The diagonal depolarization [1], best suited to depolarizers of the forms defined by Eqs. (4) or (7), and defined as

$$
\Delta_{D}=1-\frac{1}{3}\left[\sum_{i=1}^{3}\left|\left(\mathbf{m}_{\Delta}\right)_{i i}\right|\right]
$$

Both indicators vary from 0 for a non depolarizing matrix, to 1 for a perfect depolarizer.

\section{RESULTS}

\subsection{Depolarizer alone}

All the images taken with the depolarizer alone are quite similar to the top panel of Figure 3, with however, a decrease in both the image contrast and sharpness with increasing milk concentration, as seen in the top of the Figure 4 . This behavior is expected from the bulk light scattering occurring in the milk solution, as discussed above.

The values of $\Delta_{M}$ and $\Delta_{D}$ depolarization indexes measured at the image center and averaged over $10 \times 10$ pixels are plotted on the graph at the bottom of the Figure 4: these indexes are practically identical, as expected for essentially diagonal Mueller matrices.

\subsection{Depolarizer and diattenuator}

We now present the results obtained with the diattenuator placed after the depolarizer, for the same set of values of milk concentration. The diattenuation remains constant all along the series of experiments, whereas the system depolarization increases with the milk concentration.

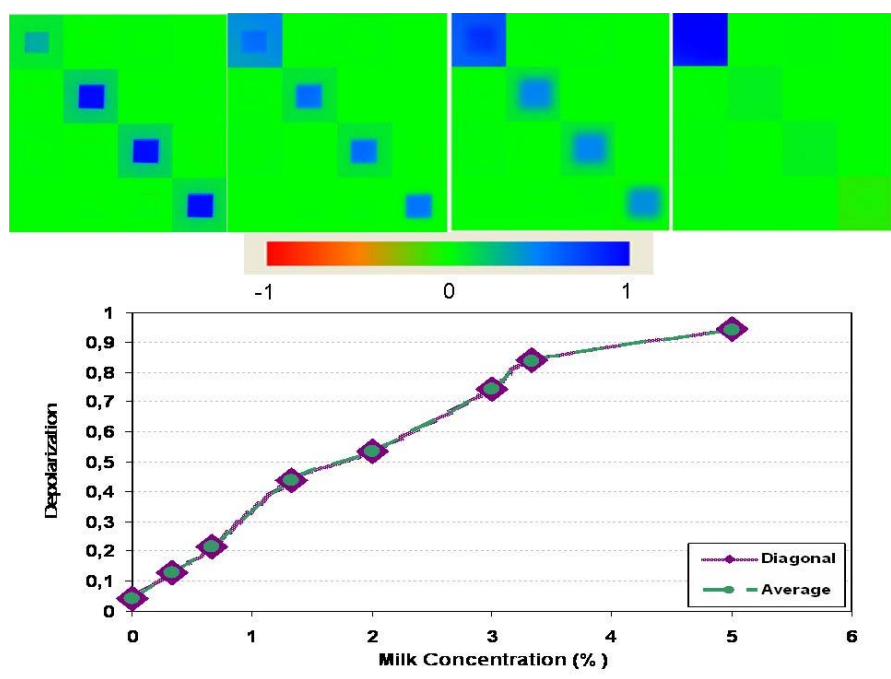

FIG. 4 Top: Mueller matrices for the depolarizer alone at milk concentrations (from left to right): $0,1.33,2$ and $5 \%$. Bottom: average and diagonal depolarization indexes at the center of the images of the depolarizer alone, for different milk concentrations. The line is a guide for the eye.
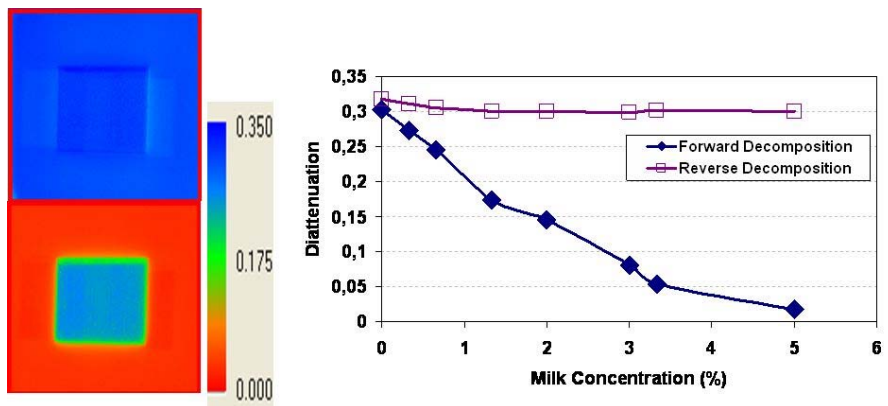

FIG. 5 Scalar diattenuation derived by forward and reverse decompositions of the images of the depolarizer followed by the diattenuator. Left: complete images, for $0.6 \%$ milk concentration. Right: scalar diattenuation at the center of the image, as a function of concentration.

The scalar diattenuation values provided by the two decompositions are summarized in Figure 5. The left panel shows the spatial variation of this diattenuation over the field of view, again for $0.6 \%$ milk concentration. While the reverse decomposition provides a uniform diattenuation as expected (except at the edges of the metal plate, where some artifacts due to the plate shadow are visible) the forward decomposition shows a sharp decrease of the diattenuation at the peripheral zone of the figure, where the depolarization is stronger. A more quantitative description of this effect is given in the right panel of the same figure, where the diattenuations provided by the two decompositions at the center of the plate are plotted versus the milk concentration.

The (erroneous !) decrease of the diattenuation provided by the forward decomposition is due to the fact that in this decomposition the diattenuator is defined from the diattenuation of the whole system, i.e. the dependence of the reflected intensity on the incident polarization. If the depolarizer is traversed before the diattenuator, the stronger the depolarization, the smaller the dependence of the overall system transmission on the incident polarization. In contrast, with the reverse decomposition the diattenuation is evaluated from the polarizance of 
the whole system, i.e. its capability to polarize an initially depolarized light. If the (diagonal) depolarizer comes first, it does not change the state of an initially depolarized light, and the polarizance is entirely due to the diattenuator set afterwards. The diattenuation evaluated from this polarizance is then independent of the depolarization, as it should.

\section{Forward Decomposition}

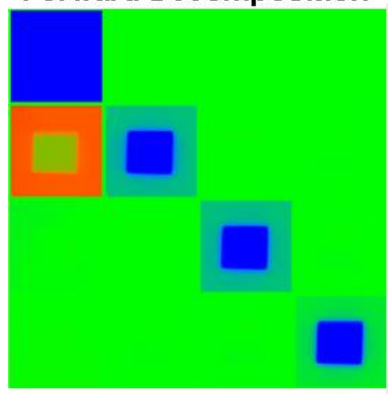

\section{Reverse Decomposition}

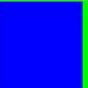

0.00000

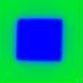

FIG. 6 Depolarization matrices $M_{\Delta}$ and $\mathbf{M}_{\Delta}^{r}$ derived from the forward (on the left) and the reverse (on the right) decompositions respectively, for the depolarizer (milk concentration $0.6 \%$ ) followed by the diattenuator. These matrices are normalized by $m_{11}$. The colorscale used is presented in the middle of the figure ( $m_{11}$ is off-scale).

We now consider the depolarization. In Figure 6 we present the $\mathbf{M}_{\Delta}$ and $\mathbf{M}_{\Delta}^{r}$ depolarization matrices (normalized by $m_{11}$ ) as derived by the two different decompositions for $0.6 \%$ milk concentration. Again, the reverse decomposition correctly reconstructs the diagonal Mueller matrix of the depolarizer alone, while the forward decomposition introduces a strong spatially inhomogeneous polarizance (term $m_{21}$ ) into $\mathbf{M}_{\Delta}$ to account for the polarizance of the whole system. However, the diagonal terms of the two reconstructed depolarizers seem quite similar, indicating that the two decompositions typically provide close values of the diagonal depolarization.

Like for the pure depolarizer, we integrate on 10x10 pixels in the center of the figure to compare the various depolarization indexes at our disposal versus milk concentration. In Figure 7 we plot the absolute values of the differences between these indexes and the depolarization measured on the milk solution alone. Concerning the diagonal depolarization $\Delta_{D}$ first, even though both decompositions provide values close to each other and to that of the pure depolarizer, the reverse decomposition is always more accurate.

The behavior of the average depolarization $\Delta_{M}$ is somewhat different. This index is the one typically used to characterize the overall depolarizing power of any input matrix $\mathbf{M}$ without any further treatment, and our results clearly indicate that for high overall depolarization values this index significantly deviates from the reference taken on the depolarizer alone. When the forward decomposition is applied, the average depolarization of the resulting $\mathbf{M}_{\Delta}$ is quite close to that of $\mathbf{M}$ : the forward decomposition does not improve the accuracy of the depolarization power characterization defined by $\Delta_{M}$. In contrast, with the reverse decomposition the index $\Delta_{M}$ evaluated with $\mathbf{M}_{\Delta}^{r}$ comes quite close to the reference values of the depolarizer alone, as it should if the decomposition is to provide new and accurate information (in this respect, we point out
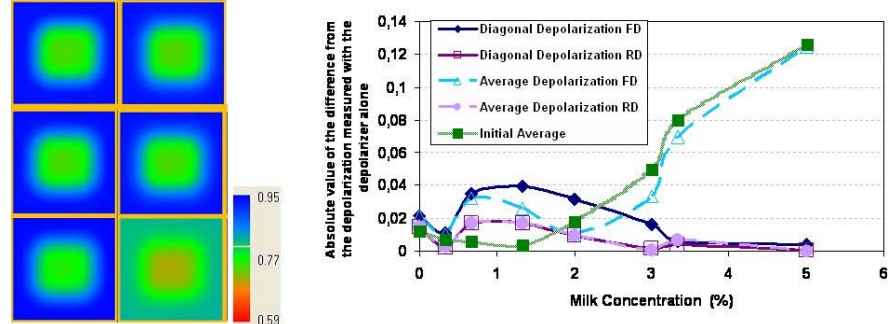

FIG. 7 Depolarization indexes provided by the two decompositions for the depolarizer followed by the diattenuator, compared to those of the depolarizer alone. Left: complete images, for $3 \%$ milk concentration: diagonal (first column) and average depolarization (second column) for the depolarizer alone (first row) and for the system of the depolarizer followed by the diattenuator as calculated by the reverse (second row) and the forward decomposition (third row). Right: variation of the absolute value of the difference of the various depolarization indexes and the one measured for the depolarizer alone with milk concentration.

that in Figure 7 the diagonal and average values of the depolarizer provided by the reverse decomposition are identical).

\subsection{Depolarizer, diattenuator and retarder}

We now present the data obtained with the three components in a row. The scalar properties of the diattenuations provided by the two decompositions remain identical to those shown in Figure 5 to within a few $10^{-3}$. The scalar retardances provided by both decompositions are remarkably constant when the depolarization varies, as expected (Figure 8). Moreover, their values are almost equal, which is an unexpected result not fully understood currently.

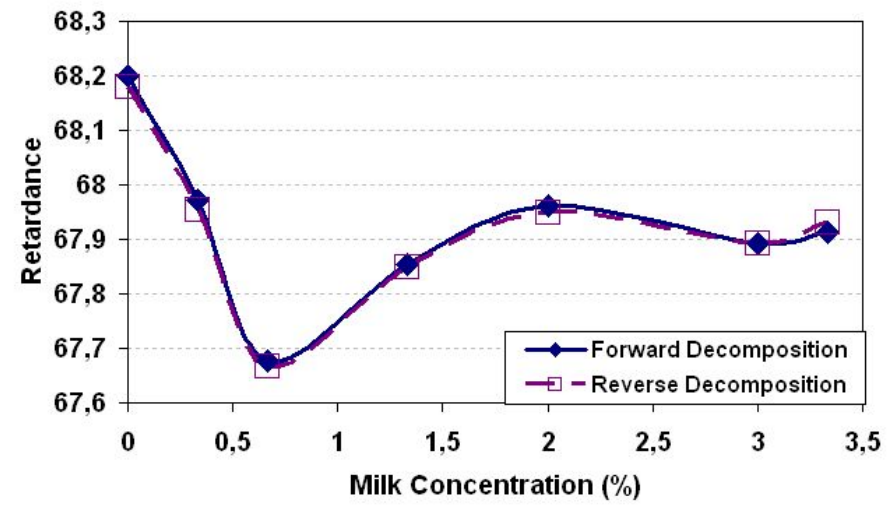

FIG. 8 Retardance for a system of a depolarizer followed by a diattenuator followed by a retarder as calculated by the forward and reverse decomposition.

The depolarization matrices provided by the two decompositions are shown in Figure 9. In this case too, only the reverse decomposition provides an essentially diagonal matrix, while the mixing of the elements in the lower $3 \times 3$ block due to the retarder is not entirely removed by the forward decomposition. In particular, the diagonal terms are clearly affected by severe errors outside the metal plate. However, all the depolarization indexes keep the same values as those obtained without the retarder (Figure 7), indicating that typical scalar quantities, such as the trace of the lower $3 \times 3$ block of the depolarizer matrices, are not affected by the retarder. This invariance is clearly a good property when one is interested only in these 
scalar quantities, but for more detailed studies, in particular those involving a physical model for the depolarizer (Rayleigh or Mie scattering regimes, for example) the scalar quantities are no longer sufficient: all the diagonal terms of the Mueller matrix of the depolarizer are needed. In such cases, the decomposition with the "wrong" order, like the forward in this study would lead to severe errors, as it can be directly seen on Figure 9.
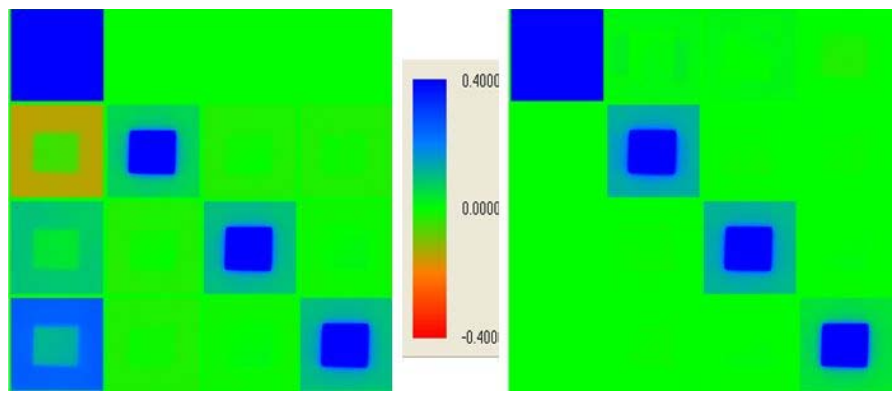

FIG. 9 Depolarizer matrices provided by the forward (left) and reverse (right) decompositions for a system of a depolarizer followed by a diattenuator, followed by a retarder.

\subsection{Biological Tissue}

We now present a first test of the forward and reverse decomposition on real tissue samples. The results of diattenuation and depolarization on a sample of pig skin are presented in Figure 10. The depolarization images ((b) and (d)) are exactly the same for both decompositions, whereas the diattenuation image derived after the application of reverse decomposition (a) reveals more details than the forward decomposition diattenuation image (c). We believe that the reverse decomposition may be more adequate for biological samples, because the skin can better be simulated by a system of a depolarizer followed by a diattenuator.

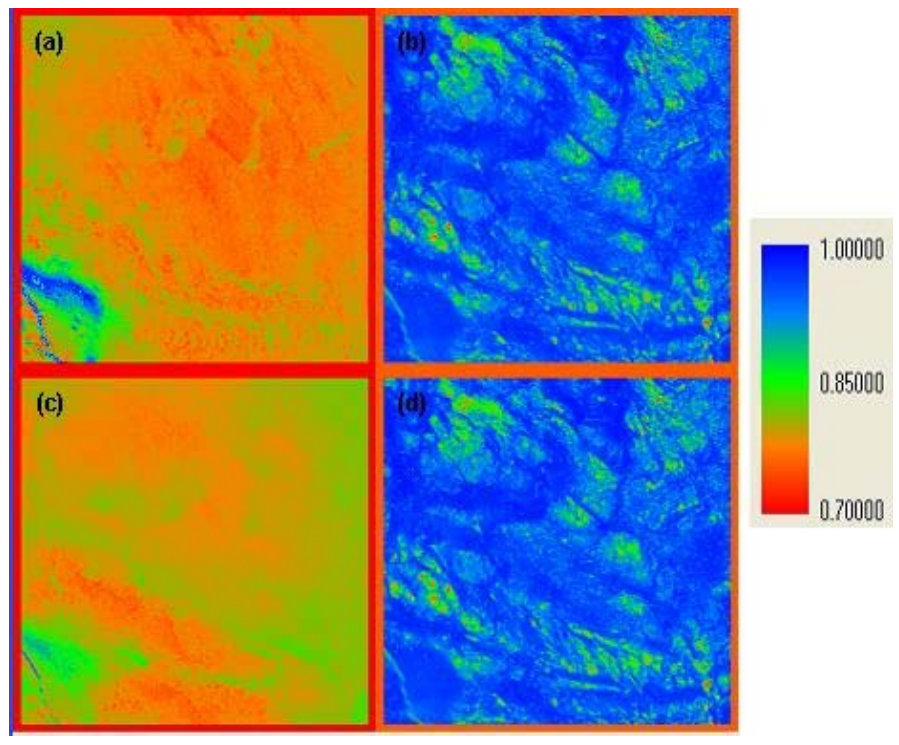

FIG. 10 Diattenuation on the left ((a) and (c)) and depolarization on the right ((b) and (d)) as calculated by reverse on the top ((a) and (b)) and forward decomposition on the bottom ((c) and (d)) on samples of pig skin.
A simple argument is illustrated in Figure 11. As a first approximation, we can consider that independently of the polarization state of the incident light what we eventually observe is a light beam that has gone under depolarization once passed the skin surface on its incidence and undergoes a diattenuation effect when it passes through the surface again on its way out. The scattering occurring in the bulk tissue, which is responsible for depolarization, also implies a loss of the spatial information about the tissue structure at the entrance side.

As a result, the diattenuation image provided by the forward decomposition is somewhat blurred. On the other hand, with the reverse decomposition the diattenuation is computed from the polarizance, due to the diattenuation of the exit face, which suffers no "blurring" due to propagation. As a result, the diattenuation image provided by the reverse decomposition is definitely sharper and conveys clearly different information about the skin structure. In this way, we may even be capable of distinguishing surface (diattenuation) from volume effects (depolarization).

For further studies and better precision we can simulate the skin by a diattenuator followed by a depolarizer followed by a diattenuator. In this way we simulate both interfaces on the way of the light in and out of the skin surface. For further details an article is being prepared by our team and it will be published soon.

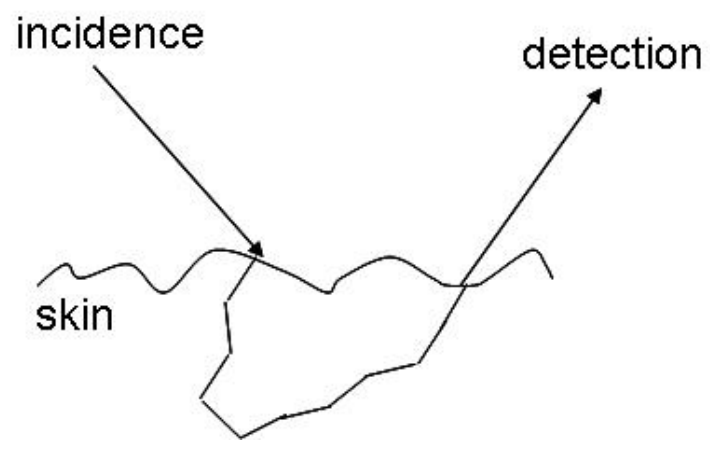

FIG. 11 Typical path of the light in a tissue.

\section{CONCLUSION}

In this study we have experimentally demonstrated the validity of the reverse polar decomposition in the case of optical systems with the depolarizer placed before the diattenuator. Contrary to the well known Lu and Chipman's forward decomposition that gives erroneous values of the scalar diattenuation for high depolarizations, the reverse decomposition achieves to retrieve the accurate values of each decomposition component (depolarization, diattenuation, retard) maintaining at the same time a diagonal depolarizer matrix. The interest of the reverse decomposition on biological applications has also been tested on real tissue samples, that we consider as a depolarizer (bulk of the tissue) followed by a diattenuator (the skin surface at light outlet). This decomposition may open new possibilities for polarimetric image treatment. 


\section{References}

[1] S. Y. Lu and R. A. Chipman, "Interpretation of Mueller matrices based on polar decomposition" J. Opt. Soc. Am. A 13, 1106-1113 (1996).

[2] J. E. Wolfe and R. A. Chipman, "Polarimetric characterization of liquid-crystal-on-silicon panels" Appl. Optics 45, 1688-1703 (2006).

[3] B. Laude-Boulesteix, A. De Martino, B. Drévillon and L. Schwartz, "Mueller polarimetric imaging system with liquid crystals" Appl. Optics 43, 2824-2832 (2004).

[4] S. Guyot, C. Vézien, B. Clairac and B. Fontas, "Optical properties filtering using Mueller's formalism: application to laser imaging" Optik 114, 289-297 (2003).
[5] J. Morio and F. Goudail, "Influence of the order of diattenuator, retarder and polarizer in polar decomposition of Mueller matrices" Opt. Lett. 29, 2234-2236 (2004).

[6] R. Ossikovski, A. De Martino and S. Guyot, "Forward and reverse product decompositions of depolarizing Mueller matrices" opt. Lett. 32, 689 (2007).

[7] A. De Martino, Y. Kim, E. Garcia-Caurel and B. Laude, “Optimized Mueller polarimeter with liquid crystals" Opt. Lett. 28, 616-618 (2003).

[8] W. I. Goldburg, "Dynamic light scattering" Am. J. Phys. 67, 1152 (1999).

[9] J. J. Gil and E. Bernabeu, "A depolarization criterion in Mueller matrices" Opt. Acta 32, 259-261 (1986). 